\title{
Collider gets yet more exotic 'to-do' list
}

\section{The Large Hadron Collider could throw up evidence of new physics earlier than expected.}

As if the Large Hadron Collider (LHC) didn't have enough to look for. It is already charged with hunting for the fabled Higgs boson, extra dimensions and supersymmetry, but physicists are now adding even more elaborate phenomena to its shopping list including vanishing dimensions that could explain the accelerating expansion of the Universe. Some argue that signs of new and exotic physics could show up in the LHC far sooner than expected.

In March, the LHC, sited at CERN, Europe's particle-physics facility near Geneva, Switzerland, began colliding protons at energies of 7 trillion electronvolts - half the final target but already three times greater than its nearest rival, the Tevatron in Batavia, Illinois. This week, particle physicists gather at the International Conference on High Energy Physics (ICHEP) in Paris to discuss what they hope to find - and when the discoveries might emerge.

Still topping physicists' wish lists is the Higgs boson, the elusive particle thought to be part of the mechanism that gives other particles their mass. If the standard model of particle physics has correctly predicted its characteristics, gathering enough data to find the Higgs should take about two more years, says Albert de Roeck, deputy spokesman for the Compact Muon Solenoid (CMS) experiment at the LHC.

But beyond the Higgs, researchers hope to see evidence of new physics. So far, accelerator experiments have repeatedly confirmed the predictions of the standard model, which encompasses all discovered particles, the Higgs and three of the fundamental forces of nature: electromagnetism; the weak force that controls radioactivity; and the strong force that binds quarks together. "It's annoying, because from a mathematical perspective, we know that the standard model must be wrong," says Greg Landsberg, a particle physicist at Brown University in Providence, Rhode Island. The model breaks down at high energies (such as those predicted in the early Universe), giving infinite answers for the strength of particle interactions, unless physicists fudge the numbers.

One addendum to the standard model that does away with this fine-tuning is supersymmetry (SUSY), which posits the existence of heavier twins for all known particles. These

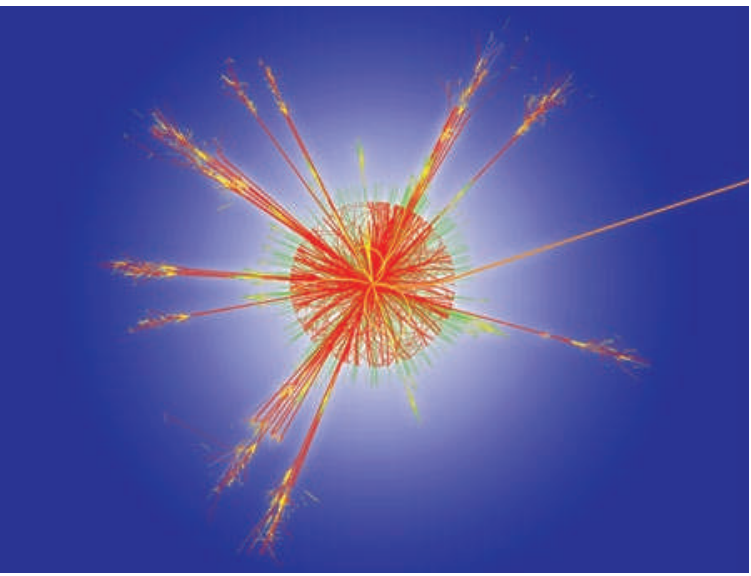

iniature black holes would decay into a spray of particles.

SUSY twins could show up at the LHC within a couple of years, says de Roeck.

The other big prize for the LHC would be evidence of extra dimensions. These are proposed by some forms of string theory, which describes the fundamental building blocks of the Universe as endlessly vibrating strands. If extra dimensions exist, their presence could show up as a shortfall in the energy of collision debris, indicating that some particles created in the smash can access these dimensions. Miniature black holes appearing during collisions could also betray the gravitational effects of these dimensions, de Roeck says.

The accelerator has already hit the high energies needed for these new particles and exotic effects to pop up. But the machine needs to ramp up its collision rate to generate enough data to prove that rare anomalies are more than statistical blips, says de Roeck.

Some argue that by focusing on SUSY and extra dimensions, physicists could miss earlier signs of new physics. Zoltan Ligeti, a physicist at the Lawrence Berkeley National Laboratory in California, and his colleagues have calculated that as it ramps up, the LHC will generate enough collisions to produce clear signatures of a hypothetical 'diquark' particle proposed by some forms of string theory. "Even with relatively little LHC data, you could show that the standard model is wrong, if you know what to look for," says Ligeti, who has a list of new particles to watch for that he will unveil at ICHEP.

Landsberg hopes to trump the standard model in grander style. He is presenting an ambitious new theory in which the number of dimensions in the Universe increases as it grows in size. He and his colleagues propose that the Universe began with just one spatial dimension and one time dimension. "Think of the Universe as a one-dimensional thread that gradually wove itself into a twodimensional tapestry as it grew, and then wrapped itself up further to create three dimensions," he says.

Reducing the spatial dimensions of the early Universe avoids the problems with the standard model, because the unwanted infinities arise only for equations describing three dimensions, says Landsberg. The team has also calculated that a fourth spatial dimension would show up as an intrinsic energy that pushes the three-dimensional Universe outwards. The effect roughly matches the acceleration of cosmic expansion, currently attributed to the mysterious 'dark energy'. "Dark energy is an echo of the fourth dimension of space," Landsberg argues.

\section{Vanishing dimensions}

The LHC could reveal "spectacular evidence" of one spatial dimension vanishing as the machine approaches the high-energy conditions seen soon after the Big Bang, says Landsberg. If his ideas are correct, then the LHC will soon start to access a two-dimensional universe. "Collision debris that you would expect to spray out in three dimensions, will instead be confined to a two-dimensional plane," he says.

Evidence of vanishing dimensions may already have been spotted in the shower of particles created by cosmic rays entering our atmosphere. De Roeck was recently approached by a team of physicists who had found some "peculiar results" while reanalysing cosmic-ray data collected 15 years ago in the Pamir mountains in central Asia. "Instead of particle jets spraying everywhere, as expected, the jets were oddly aligned in a way that can't be explained by conventional models," says de Roeck. The CMS collaboration is already planning to see if the effect can be reproduced at the LHC (M. Deile et al. http://arxiv.org/abs/1002.3527v1; 2010).

"Landsberg's model remains highly speculative, but it may just be that the signature for it has been seen," says de Roeck. "That's something we certainly want to try to confirm or rule out at the LHC."

Zeeya Merali 